\title{
Plus le temps passe, plus les conditions d'incarcération empirent Francis Beauchamp
}

$\mathbf{B}_{\text {problème je voudrais faire part de mon insatisfaction envers plusieurs }}^{\text {onjour }}$ services correctionnels.

\section{SALAIRE}

J'ai débuté ma sentence fédérale en 2003. À bien y repenser, je trouve qu'on était mieux dans ce temps-là, alors qu'il y avait plus de respect de la part des gardiens. Nous avions un salaire de 6,90\$/jour, les bonus y étaient ajoutés. Dans certains emplois, on avait plusieurs grades (A, B, C et D), alors qu'aujourd'hui, il y a seulement le grade C et D. Ainsi, dans les grades dits " Médium », nous faisions $69 \$$ chaque deux semaines. De cette manière, nous étions capables de mettre un peu d'argent de côté dans un compte personnel en prévision de notre sortie de l'institution. De plus, nous étions mieux préparés à faire notre entrée dans le monde extérieur, car avec cet argent supplémentaire nous pouvions acheter des vêtements de travail, des bottes d'hiver, des billets d'autobus et finalement nous chercher un emploi sans avoir de tracas. Aujourd'hui, je vis déjà un 'stress' sachant que je n'aurai aucun moyen ni argent afin de réussir ma réinsertion, car j'y suis maintenant au deux tiers de ma sentence (libération conditionnelle). Le système a bien changé depuis, auparavant, l'institution avait les moyens de me diriger vers des ressources recouvrant l'aide à l'emploi, le logement et bien d'autres services. Toutefois, aujourd'hui ce n'est plus pareil. Tous, nous quittons la prison/pénitencier, tel un individu sans-abri. Cela mène plusieurs d'entre nous à retrouver leurs anciens comportements, car ils sont laissés à eux-mêmes.

\section{OPTOMÉTRISTE}

Cela fait déjà 5 mois que j'attends afin de voir un optométriste. J'ai effectué des requêtes tous les mois, car je trouve le délai afin d'obtenir un rendezvous comme étant non raisonnable et respectueux de l'individu. La seule réponse que j'ai pu obtenir se limite à m'indiquer que « je suis sur la liste d'attente ». À l'heure actuelle, je suis dans l'obligation de porter les lunettes d'un autre prisonnier lorsque je dois remplir des papiers. Je sens que le 
fait que je ne porte pas des lunettes ajustées à ma vue brise celle-ci peu à peu. Toutefois, je ne suis pas le seul détenu dans cette situation. Certains d'entre eux sont sur la liste d'attente depuis 12 à 24 mois afin d'obtenir un examen de la vue. Je trouve donc cette situation inconcevable. En 2013, j'avais passé un examen de la vue et je me rappelle que l'attente pour celuici n'était que de 30 jours. Ainsi, il est facile de conclure que le système ne fait qu'empirer et se compliquer. Je ne peux comprendre qu'aujourd'hui ce système ne fait que régresser au lieu de s'améliorer. À tous ceux impliqués dans la construction et le maintien de ce système, je suis profondément déçu de sa mise en place présentement. Comment oseriez-vous me dire que ces délais (12-24 mois) sont concevables et 'normal' dans le cas d'un examen de la vue? Pouvez-vous imaginer le temps que cela va prendre afin d'obtenir une prescription et des lunettes de vue?

\section{DENTUROLOGIE}

Malheureusement, la même situation se dessine pour ce qui est d'obtenir un rendez-vous avec un dentiste. Dans mon cas, j'aurais besoin d'un partiel afin d'être en mesure de manger confortablement. L'histoire se réécrit alors qu'après quatre requêtes, le service de denturologie m'affirme à chacune d'elles que je suis sur une liste d'attente et que ces délais sont acceptables considérant les demandes. Présentement, j'empire ma situation dentaire à chaque. Cette situation n'est pas normale et je trouve que l'on s'occupe mal de nous. Je ressens donc que je ne suis qu'un simple numéro parmi tant d'autres.

\section{LA NOURRITURE}

Je ne passerai pas par quatre chemins : ce n'est pas mangeable! Tout d'abord, les portions des repas sont trop petites, tel un menu pour enfant. Nous sommes des hommes d'âge adulte, nous avons donc un grand appétit et celle-ci est constante. Ainsi, une à deux heures suivant notre repas, je saute déjà dans le pain, très souvent sec lorsqu'on le reçoit. On ne mange donc pas à notre faim, alors qu'auparavant, il n'y avait pas de problème de ce côté. Maintenant, on nous sert des salades composées de la laitue seulement, une pomme ou un autre désert qui n'est pas toujours très bon. Bref, le plus important à retenir est les petites portions qui nous sont offertes. 


\section{ACTIVITÉS}

Tout d'abord, nous n'avons plus d'activités comme auparavant pour nous (ré)intégrer dans la société. Par exemple, il y a quelques années, nous avions quatre visites par année auprès d'enfants atteints d'un ou plusieurs handicapés. Je trouve que cette activité nous permettait une grande réflexion à plusieurs niveaux et dans mon cas ces types d'activités me font grandir dans mon cheminement personnel. De plus, il y avait des bénévoles qui venaient jouer quelques matchs de volleyball contre nous. Tout le monde trouvait cela bien amusant et nous permettait de côtoyer des individus provenant de l'extérieur de l'institut.

\section{APPEL POUR UNE MOBILISATION VERS UNE RÉFORME DU SYSTÈME}

Tous ceux qui seront en mesure de lire cette publication devront débuter la rédaction de lettres concernant la réforme du système. Celles-ci devraient être envoyées à leur député fédéral, le ministre de la sécurité publique pour le parti libéral, Bill Blair. Finalement, il est bien évident que le premier ministre du Canada, Justin Trudeau, doit être impliqué afin d'obtenir cette mobilisation. De plus, un ou deux sénateurs de votre province doivent recevoir ces lettres afin d'avoir un grand impact. Les prisonniers devraient avoir accès à ce genre d'évènement chaque semaine, car il ne s'agit pas d'une activité dispendieuse. La distribution de lettres au Parlement est gratuite, car les étampes ne sont pas obligatoires. Dans ce cas, l'utilisation d'un ordinateur serait intéressante, car les mêmes lettres pourraient être envoyées aux identifiants ci-haut, tout en y changeant les titres. Ainsi, les individus pouvant apporter des changements au système recevront ces lettres de façon hebdomadaire. Tout ce dont ils ont besoin c'est la bonne motivation pour faire avancer les choses. La réception de multiples lettres de complaintes

concernant les mêmes problèmes et cela toutes les semaines est nécessaire afin de guider ces personnes d'importances. Donc, il est important que vous fassiez votre part afin de forcer le changement.

Voici plusieurs sujets de rédaction :

-Le monde carcéral

-Les formations professionnelles 
-L'alimentation dans les institutions

-La construction de maisons de transition-financement des sentences à perpétuité

-Les opportunités d'emploi pour les individus incarcérés après 25 ans

-Le trop grand pouvoir que détiennent les agents de libération conditionnelle

Vous pouvez envoyer vos lettres dans une enveloppe scellée contenant ces informations :

« Le nom du destinataire »

Bâtiment parlementaire

Ottawa, ON

KIA 0A4

\section{À PROPOS DE L'AUTEUR}

Francis Beauchamp est un prisonnier à l'Établissement Archambault. 